05

\title{
Расчет эффективных параметров термоэлектромагнитоупругих слоистых сред
}

\author{
() А.С. Старков, И.В. Кудрявцева, К.А. Старков, К.В. Корзенков
}

Университет ИТМО,

191002 Санкт-Петербург, Россия

e-mail: ferroelectrics@ya.ru

(Поступило в Редакцию 7 июня 2016 г.)

\begin{abstract}
Проведено усреднение системы слоистых мультиферроиков с учетом тепловых свойств слоев. Для получения эффективных характеристик системы используется матричный метод усреднения. Получены формулы, описывающие влияние магнитоэлектроупругих коэффициентов слоев на тепловые характеристики. Показана возможность появления пирокоэффициентов в системе даже при их отсутствии в каждом слое. Из-за взаимодействия полей теплоемкость системы перестает быть аддитивной величиной и становится зависящей от диэлектрической и магнитной проницаемостей слоев.
\end{abstract}

DOI: 10.21883/JTF.2017.07.44673.1921

\section{Введение}

Среди имеющихся в настоящее время методов получения усредненных (эффективных) характеристик композитных сред наиболее обоснованными с математической точки зрения являются метод усреднения периодических сред $[1,2]$ и матричный метод усреднения слоистых сред (ММУ) [3,4]. Для слоистых структур оба метода приводят к одинаковым результатам. При усреднении периодических сред для получения эффективных значений достаточно решить исходные дифференциальные уравнения в пределах одной элементарной ячейки, а не во всем пространстве. Для ММУ решать какие-то ни было дифференциальные уравнения нет необходимости. Все операции сводятся к действиям над матрицами. В этом и состоит основное преимущество ММУ над другими методами. Еще одним достоинством ММУ является возможность достаточно просто вычислять поправки к уже найденным значениям. Его превосходство становится неоспоримым, если рассматривается усреднение слоистой среды, описываемой множеством физических параметров. Такими средами являются мультиферроики - одни из наиболее перспективных материалов для использования в новейших технологических устройствах. Данные материалы позволяют управлять одним из физических полей (электрическим, магнитным или упругим) при помощи другого [5,6]. В естественных мультиферроиках взаимодействие электрического и магнитного полей весьма мало, поэтому для усиления магнитоэлектрического эффекта было предложено использовать искусственные мультиферроики, в которых связь указанных полей осуществляется посредством упругого поля $[7,8]$. Наиболее простыми искусственными мультиферроиками являются слоистые структуры, каждый слой в которых является пьезоэлектриком или пьезомагнетиком. Если к трем вышеуказанным полям добавить еще тепловое поле, то попарные взаимодействия могут усилиться [9], но расчет распределения всех полей в слоистой структуре требует решения существенно более сложной системы дифференциальных уравнений. Одних переменных для описания полей нужно более 20 . Цель настоящей работы - нахождение явных формул для эффективных характеристик слоев мультиферроиков с учетом их теплового взаимодействия. Ранее эти характеристики получались только в результате численных расчетов [10] либо в статическом приближении [9]. Так как ММУ не накладывает никаких ограничений на физические величины, то результаты работы могут быть использованы при расчете эффективных параметров метаматериалов [11]. К сожалению, в настоящее время ММУ применим только для нахождения свойств слоистых систем. В случае произвольной геометрии расположения фаз в композитном материале следует использовать более сложные методы $[1,12]$.

\section{Матричный метод усреднения}

Вначале приведем основные положения ММУ для слоистой среды, состоящей из $n$ слоев (рис. 1). В системе координат $(x, y, z)$ (не обязательно декартовой) эта среда занимает область $0<z<L$, а границы слоев задаются уравнениями $l_{i}<z<l_{i+1}, i=1,2, \ldots, n$, $l_{0}=0, l_{n+1}=L$. Толщину слоя с номером $i$ обозначим через $h_{i}=l_{i+1}-l_{i},\left(h_{1}+h_{2}+\ldots h_{n}=L\right)$, а относительную толщину - через $\vartheta_{i}=h_{i} / L$. В дальнейшем индекс $i$,

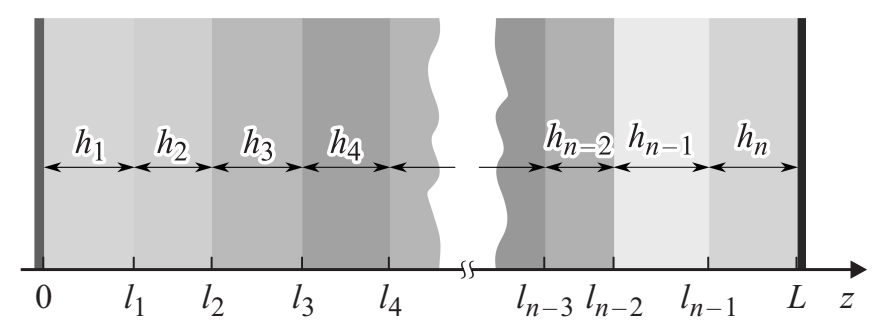

Рис. 1. Конфигурация слоистой системы. 
указывающий на принадлежность величины к слою с этим номером, будем опускать, если это не вызовет неоднозначных толкований. Основная идея матричного метода усреднения заключается в том, что система уравнений, описывающих свойства слоя, должна иметь вид

$$
\frac{\partial U}{\partial z}=A U+F, \quad V=B U
$$

Здесь $\{A, B\}$ - операторные матрицы, характеризующие материальные свойства слоя, $\{U, V\}-$ столбцы неизвестных, $F-$ столбец внешних сил. Для каждого слоя матрицы $A, B$ и столбцы $F$ свои: $A=A_{i}(z)$, $B=B_{i}(z), F=F_{i}(z)$ в слое с номером $i$. Кроме того, матрицы $A$ и $B$ могут зависеть от переменных $\{x, y\}$ и производных по ним и по времени $t$. В вектор-столбец переменных $U$ входят все те величины, которые непрерывны на границах раздела различных слоев. Элементы столбца $V$ на границах слоев могут испытывать разрыв. В дальнейшем будем рассматривать более простой случай, когда внутри каждого слоя матрицы $A_{i}$ и $B_{i}$ не зависят от $z$. В противном случае в последующих формулах достаточно произвести замену

$$
A_{i} h_{i} \rightarrow \int_{l_{i}}^{l_{i+1}} A_{i}(z) d z
$$

Усреднение линейных сред не должно зависеть от внешних сил, поэтому можно усреднять матрицы $A, B$ и столбцы $F$ независимо друг от друга. Решение системы уравнений (1) при $F=0$ в слое с номером $i$ выражается через значение $U(z)$ на границе при помощи матричнооператорной экспоненты $[3,4]$

$$
U(z)=\exp \left(A_{i} z\right) U\left(l_{i-1}\right) .
$$

Следовательно, значения $U(z)$ на внешних границах связаны при помощи упорядоченного произведения экспонент

$$
U(h)=\exp \left(A_{n} h_{n}\right) \exp \left(A_{n-1} h_{n-1}\right) \ldots \exp \left(A_{1} h_{1}\right) U(0) .
$$

Если заменить исходную многослойную систему на эффективный слой той же толщины $L$, описываемый системой уравнений

$$
\frac{\partial X}{\partial z}=A^{\mathrm{ef}} X, \quad Y=B^{\mathrm{ef}} X,
$$

то для него связь решений на внешних границах задается соотношением

$$
U(h)=\exp \left(A^{\mathrm{ef}} h\right) U(0) .
$$

Сравнивая (4) и (6), получаем уравнение для определения эффективных значений матрицы $A^{\mathrm{ef}}$

$$
A^{\text {ef }}=\frac{1}{h} \ln \left(\exp \left(A_{n} h_{n}\right) \exp \left(A_{n-1} h_{n-1}\right) \ldots \exp \left(A_{1} h_{1}\right)\right) .
$$

Уравнение (7) является точным, но для получения $A^{\mathrm{ef}}$ нет смысла его использовать, так как каждая матрица $A_{i}$ не является постоянной и может содержать производные по независимым переменным $x, y, t$. Ввиду этого в [4] было предложено воспользоваться разложением Кэмпбелла-Хаусдорфа [13], которое в рассматриваемом случае имеет вид

$$
A^{\mathrm{ef}}=\sum_{i=1}^{n} A_{i} \vartheta_{i}+\frac{h}{2} \sum_{i>j}\left[A_{i}, A_{j}\right] \vartheta_{i} \vartheta_{j}+\ldots
$$

Здесь $\left[A_{i}, A_{j}\right]=A_{i} A_{j}-A_{j} A_{i}$ есть коммутатор $A_{i}$ и $A_{j}$. Ряд (8) есть формальный ряд по степеням толщины всей системы $h$. Первый невыписанный член ряда содержит коммутаторы вида $\left[A_{i},\left[A_{j}, A_{k}\right]\right]$. Если ограничиться только первой суммой в $(8)$, то получаем основную формулу MMY

$$
A^{\mathrm{ef}}=\sum_{1}^{n} A_{i} \vartheta_{i} \equiv \bar{A}, \quad B^{\mathrm{ef}}=\bar{B}, \quad F^{\mathrm{ef}}=\bar{F} .
$$

Если включать в матрицу $A^{\mathrm{ef}}$ следующие члены ряда (8), то мы будем получать все более точные значения ее элементов. К сожалению, при этом может быстро расти порядок дифференциальных уравнений, описывающих эффективную среду. Помимо этого может меняться сам характер уравнений, появляться дополнительные токи, заряды, силы и т.п. Ввиду этого в настоящей работе ограничимся только выписанными в (8) членами. На последнем шаге ММУ осушествляется обратный переход к исходным уравнениям с усредненными коэффициентами. Несмотря на простоту формул (9), для эффективных значений физических параметров могут получаться весьма сложные выражения даже в обычной теории упругости [3,4].

Слои в ММУ могут иметь произвольную форму: плоскопараллельные [4], цилиндрические, сферические [14], эллипсоидальные, но в настоящей работе рассмотрим только плоскопараллельные слои.

\section{Постановка задачи}

Рассмотрим систему плоскопараллельных термоэлектромагнитоупругих слоев. Толщину всей системы $L$ считаем малой как по сравнению с длинами всевозможных волн, которые могут в ней распространяться, так и по сравнению с характерным размером неоднородностей внешних полей вдоль слоев [4]. Для описания распределения полей в каждом слое будем использовать отклонение температуры $T$ от температуры окружающей среды $T_{0}$, напряженности электрического и магнитного полей $E_{i}$ и $H_{i}$, вектора электрической и магнитной индукции $D_{i}$ и $B_{i}$, смещений $u_{i}$, тензора деформаций $u_{i k}=\left(u_{i, k}+u_{k, i}\right) / 2$ и напряжений $\sigma_{i k}$. Здесь и далее индекс после запятой означает дифференцирование по соответствующей переменной $X_{, i}=\partial X / \partial x_{i}, x_{1}=$ $=x, x_{2}=y, x_{3}=z$, а точка над величиной - производную по времени. Каждый слой описывается плотностью $\rho$, объемной теплоемкостью $C$, коэффициентами 
теплопроводности $\lambda_{i k}$, упругими постоянными $c_{i k l m}$, пьезоэлектрическими и пьезомагнитными модулями $e_{i k l}$ и $f_{i k l}$, диэлектрическими и магнитными проницаемостями $\varepsilon_{i k}$ и $\mu_{i k}$, магнитоэлектрическими коэффициентами $g_{i k}$, пироэлектрическими и пиромагнитными коэффициентами $p_{i}$ и $q_{i}$, термоупругими модулями $\beta_{i k}$. Термоупругие модули также будем называть пироупругими коэффициентами. Для упрощения последующих формул дальнейшие вычисления проведем для случая трансверсально изотропной среды, когда ось симметрии совпадает с осью $z=x_{3}$ и направлена поперек слоев. В этом случае для описания каждого слоя имеется 2 коэффициента теплопроводности $\left\{\lambda_{1}, \lambda_{3}\right\}$, по 2 пироэлектрических $\left\{p_{1}, p_{3}\right\}$, пиромагнитных $\left\{q_{1}, q_{2}\right\}$ и пироупругих $\left\{\beta_{1}, \beta_{3}\right\}$ коэффициента, 2 диэлектрические $\left\{\varepsilon_{1}, \varepsilon_{3}\right\}$ и 2 магнитные $\left\{\mu_{1}, \mu_{3}\right\}$ проницаемости, 2 магнитоэлектрических коэффициента $\left\{g_{1}, g_{3}\right\}, 3$ пьезоэлектрических $\left\{e_{31}, e_{15}, e_{33}\right\}$ и 3 пьезомагнитных $\left\{f_{31}, f_{15}, f_{33}\right\}$ модуля, а также 5 упругих постоянных $\left\{c_{11}, c_{12}, c_{13}, c_{33}, c_{44}\right\}$. Здесь при записи индексов осуществлен переход к обозначениям Фойгта. Заметим, что ММУ применим при произвольной анизотропии слоев, но тогда существенно возрастает порядок обращаемых матриц и количество материальных постоянных. Так что ограничимся аксиально симметричным случаем, когда каждый слой характеризуется 27 материальными постоянными. Цель дальнейшего определение значений этих 27 постоянных для эффективного слоя. Полученные значения будем снабжать индексом ef.

Уравнения, описывающие систему в линейном приближении, имеют вид $[15,16]$

$$
\begin{gathered}
C \dot{T}=\lambda_{i k} T_{, i k}+Q+T_{0}\left(\beta_{i k} \dot{u}_{i k}-p_{i} \dot{E}_{i}-q_{i} \dot{H}_{i}\right), \\
\sigma_{i k, k}+F_{i}=\rho \ddot{u}_{i}
\end{gathered}
$$

$\operatorname{rot} \mathbf{H}=\mathbf{j}+\dot{\mathbf{D}}, \quad \operatorname{rot} \mathbf{E}=-\mathbf{B}, D_{i, i}=q, B_{i, i}=0$,

где $q$ - объемная плотность зарядов, $F_{i}$ - вектор внешних сил, $Q-$ плотность тепловых источников. При записи (10) использовано соглашение Эйнштейна: по повторяющимся индексам производится суммирование. Последняя пара уравнений Максвелла в (10) является следствием первых двух и условием согласования этих уравнений является равенство $j_{i, i}=-\dot{q}$. Материальные соотношения записываются в виде

$$
\begin{gathered}
\sigma_{i k}=c_{i k l m} u_{k l}-e_{i k l} E_{k}-f_{i k l} H_{k}-\beta_{i k} T, \\
D_{i}=\varepsilon_{i k} E_{i}+g_{i k} H_{k}+e_{k l i} u_{k l}+p_{i} T, \\
B_{i}=g_{i k}+\mu_{i k} H_{k}+f_{k l i} u_{k k}+q_{i} T .
\end{gathered}
$$

Для корректной постановки задачи о расчете термоэлектромагнитоупругого поля выписанные уравнения следует дополнить начальными условиями и условиями на внешних границах. Можно проверить, что усреднение величин, входящих в начальные условия, осуществляется по тем же правилам, что и усреднение этих величин в уравнениях. Граничные условия на внешних границах скажутся только при постановке задачи для эффективного слоя. Ввиду этого ни начальные, ни граничные условия на внешних границах не выписываем.

\section{Преобразование уравнений}

Согласно ММУ составим из величин, непрерывных на границах внутренних слоев, столбец $U$ из 14 переменных

$U=\left(T, J_{3}, u_{1}, u_{2}, u_{3}, \sigma_{13}, \sigma_{23}, \sigma_{33}, E_{1}, E_{2}, D_{3}, H_{1}, H_{2}, B_{3}\right)^{\mathrm{tr}}$,

где $J_{k}=\lambda_{i k} T_{, i}-$ есть тепловой поток вдоль координаты $x_{k}$, а индекс $\operatorname{tr}$ означает транспонирование. Граничные условия на внутренних границах как раз и заключаются в непрерывности столбца $U$. Отметим, что величины $D_{3}, B_{3}$ можно не включать в столбец $U$, так как их непрерывность на границах раздела различных сред вытекает из непрерывности тангенциальных компонент напряженностей электрического и магнитного полей $E_{1}$, $E_{2}, H_{1}, H_{2}$. Оставшиеся 11 переменных включим в столбец $V$ :

$$
V=\left(\sigma_{11}, \sigma_{22}, \sigma_{22}, J_{1}, J_{2}, D_{1}, D_{2}, E_{3}, B_{1}, B_{2}, H_{3}\right)^{\operatorname{tr}} .
$$

После определения столбцов $U$ и $V$ исходную систему уравнений (10),(11) в ММУ следует преобразовать к виду (1). Процесс получения матриц $A, B$ и $F$ достаточно простой, но громоздкий. В более простых случаях он подробно описан в $[3,4,14]$. Первым результатом усреднения $A, B$ и $F$ являются физически очевидные формулы для эффективной плотности и внешней силы

$$
p^{\mathrm{ef}}=\bar{\rho} \equiv \sum_{k=1}^{n} \vartheta_{k} \rho_{k}, \quad F_{i}^{\mathrm{ef}}=\bar{F}_{i} .
$$

Усреднение оставшихся величин рассмотрим более подробно. Выпишем уравнения (10) (без уравнений на тензор напряжений) в развернутом виде. Начнем с уравнения теплопроводности

$$
\begin{aligned}
& C \dot{T}=\lambda_{1}\left(T_{, 11}+T_{, 22}\right)+J_{3,3}+T_{0}\left[\beta_{3} \dot{u}_{33}+p_{3} \dot{E}_{3}+q_{3} \dot{H}_{3}\right. \\
& \left.+\beta_{1}\left(\dot{u}_{11}+\dot{u}_{22}\right)+p_{1}\left(\dot{E}_{1}+\dot{E}_{2}\right)+q_{1}\left(\dot{H}_{1}+\dot{H}_{2}\right)\right] .
\end{aligned}
$$

В уравнениях Максвелла ограничимся только теми уравнениями, которые содержат производные по $z$ от переменных столбца $U$ :

$$
\begin{gathered}
E_{3,2}-E_{2,3}=-\dot{B}_{1}, \quad E_{1,3}-E_{3,1}=-\dot{B}_{2}, \\
D_{1,1}+D_{2,2}+D_{3,3}=q, \quad H_{3,2}-H_{2,3}=\dot{D}_{1}+j_{1}, \\
H_{1,3}-H_{3,1}=\dot{D}_{2}+j_{2}, \quad B_{1,1}+B_{2,2}+B_{3,3}=0
\end{gathered}
$$

Материальные соотношения (11) запишем в виде

$$
\begin{array}{rr}
\sigma_{11}=c_{11} u_{1,1}+c_{12} u_{2,2}+c_{13} u_{3,3}-e_{31} E_{3}-f_{31} H_{3}- & \beta_{1} T, \\
& (17 \mathrm{a}) \\
\sigma_{22}=c_{12} u_{1,1}+c_{11} u_{2,2}+c_{13} u_{3,3}-e_{31} E_{3}-f_{31} H_{3}- & \beta_{1} T, \\
& (17 \mathrm{~b}) \\
\sigma_{33}=c_{13} u_{1,1}+c_{13} u_{2,2}+c_{33} u_{3,3}-e_{33} E_{3}-f_{33} H_{3} & -\beta_{3} T, \\
\sigma_{23}=c_{44}\left(u_{2,3}+u_{3,2}\right)-e_{15} E_{2}-f_{15} H_{2}, & (17 \mathrm{c})
\end{array}
$$




$$
\begin{gathered}
\sigma_{13}=c_{44}\left(u_{1,3}+u_{3,1}\right)-e_{15} E_{1}-f_{15} H_{2}, \\
\sigma_{12}=c_{66}\left(u_{1,2}+u_{2,1}\right), \\
D_{1}=e_{15}\left(u_{1,3}+u_{3,1}\right)+\varepsilon_{1} E_{1}-g_{1} H_{1}, \\
D_{2}=e_{15}\left(u_{2,3}+u_{3,2}\right)+\varepsilon_{1} E_{2}-g_{1} H_{2}, \\
D_{3}=e_{31}\left(u_{1,1}+u_{2,2}\right)+e_{33} u_{3,3}+\varepsilon_{3} E_{3}+g_{3} H_{3}, \\
B_{1}=f_{15}\left(u_{1,3}+u_{3,1}\right)+g_{1} E_{1}+\mu_{1} H_{1}, \\
B_{2}=f_{15}\left(u_{2,3}+u_{3,2}\right)+g_{1} E_{2}+\mu_{1} H_{2}, \\
B_{3}=f_{31}\left(u_{1,1}+u_{2,2}\right)+f_{33} u_{3,3}+g_{3} E_{3}+\mu_{3} H_{, 3},
\end{gathered}
$$

где, как обычно, $c_{66}=\left(c_{11}-c_{12}\right) / 2$. Уравнения Максвелла (16) после переноса производных по выделенной координате в левую часть позволяют выписать шесть последних строчек матрицы $A$. Видно, что коэффициенты перед неизвестными в этих матрицах не зависят от номера слоя. А меняются только ток и плотность электрических зарядов. Значит,

$$
\mathbf{j}^{\mathrm{ef}}=\overline{\mathbf{j}}, \quad q^{\mathrm{ef}}=\bar{q} .
$$

В материальных уравнениях коэффициенты в уравнениях (17f)-(17h), (17j), (17k) задают соответствующие строки матрицы $B$. Уравнения (17a), (17b) содержат производную по $x_{3}\left(u_{33}\right)$ и величины $E_{3}, H_{3}$, которые могут быть разрывны на границах. Ввиду этого для приведения этих уравнений к требуемому виду необходимо исключить перечисленные величины. Для этого обратимся к уравнениям, которые их содержат:

$\left\{\begin{array}{l}c_{33} u_{3,3}-c_{33} E_{z}-f_{33} H_{z}=\sigma_{33}-c_{13}\left(u_{11}+u_{22}\right)+\beta_{3} T, \\ e_{33} u_{3,3}+\varepsilon_{3} E_{z}+g_{3} H_{z}=D_{3}-e_{31}\left(u_{11}+u_{22}\right)-p_{3} T, \\ f_{33} u_{3,3}+g_{3} E_{z}+\mu_{3} H_{z}=B_{3}-f_{31}\left(u_{11}+u_{22}\right)-q_{3} T .\end{array}\right.$

Перейдем в (19) к матричной записи. Для этого введем

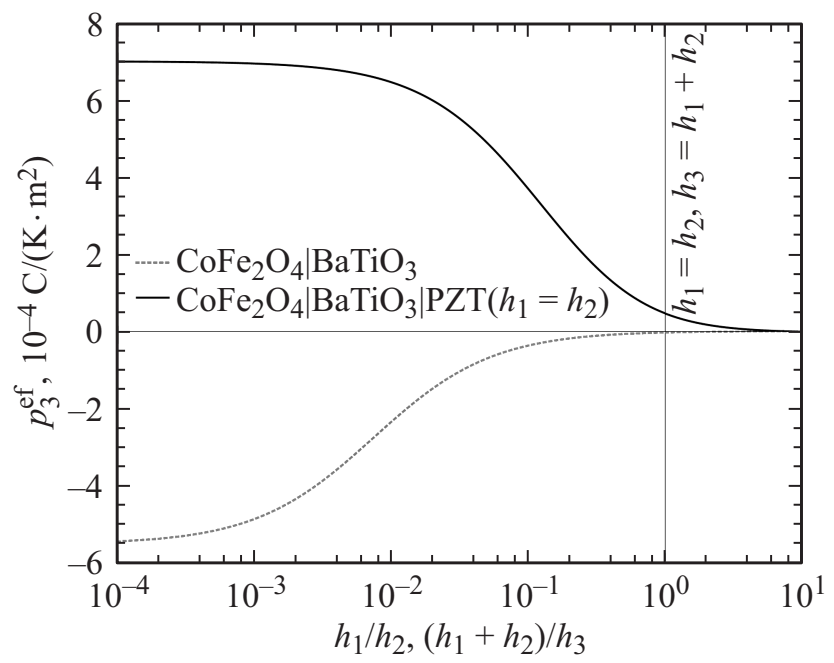

Рис. 2. Зависимость пироэлектрического коэффициента от отношений толщин слоев для двух- и трехслойной систем.

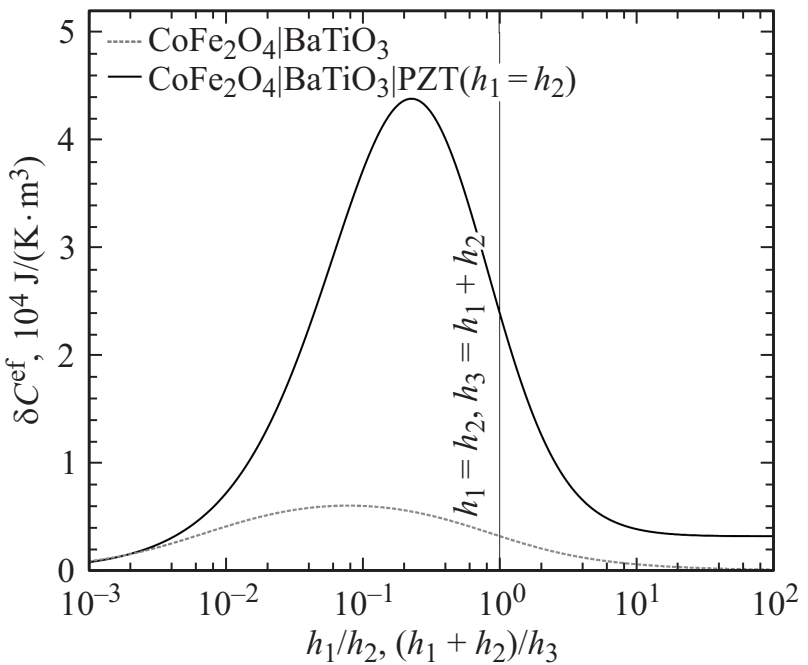

Рис. 3. Зависимость неаддитивной части теплоемкости от отношений толщин слоев для двух- и трехслойной систем.

матрицу $Q$ и столбцы $\Phi, \Psi, P, R$ следуюшим образом:

$$
\begin{gathered}
Q=\left(\begin{array}{ccc}
c_{33} & -e_{33} & -f_{33} \\
e_{33} & \varepsilon_{3} & g_{3} \\
f_{33} & g_{3} & \mu_{3}
\end{array}\right), \quad \Phi=\left(\begin{array}{c}
u_{3,3} \\
E_{3} \\
H_{3}
\end{array}\right), \\
\Psi=\left(\begin{array}{c}
\sigma_{33} \\
D_{3} \\
B_{3}
\end{array}\right), R=\left(\begin{array}{c}
c_{13} \\
e_{31} \\
f_{31}
\end{array}\right), \quad P=\left(\begin{array}{c}
\beta_{3} \\
-p_{3} \\
-q_{3}
\end{array}\right) .
\end{gathered}
$$

После решения системы (19) получаем 3 строчки для матриц $A$ и $B$

$$
\Phi=Q^{-1} \Psi-Q^{-1} R\left(u_{11}+u_{22}\right)+Q^{-1} P T .
$$

Для эффективного слоя эти строчки должны иметь вид

$$
\Phi=\left(Q^{\mathrm{ef}}\right)^{-1} \Psi-\left(Q^{\mathrm{ef}}\right)^{-1} R^{\mathrm{ef}}\left(u_{11}+u_{12}\right)+\left(Q^{\mathrm{ef}}\right)^{-1} P^{\mathrm{ef}} T .
$$

Сравнение уравнений (21) и (22) дает возможность выписать усредненные значения введенных матриц и столбцов

$$
\begin{aligned}
& Q^{\text {ef }}=\left(\overline{Q^{-1}}\right)^{-1}, \\
& R^{\text {ef }}=Q^{\text {ef }} \overline{Q^{-1} R}, \\
& P^{\text {ef }}=Q^{\text {ef }} \overline{Q^{-1} P} .
\end{aligned}
$$

После подстановки (18) в (23) получаем эффективные значения тепловых коэффициентов

$$
\begin{gathered}
p_{1}^{\mathrm{eff}}=\overline{p_{1}}, \quad q_{1}^{\mathrm{eff}}=\overline{q_{1}}, \quad C^{\mathrm{eff}}=\bar{C}+\delta C, \\
\delta C=T_{0}\left[\left(P^{\mathrm{eff}}\right)^{\mathrm{tr}} \overline{Q^{-1} P}-\overline{P^{\mathrm{tr}} Q^{-1} P}\right], \\
\beta_{1}^{\mathrm{eff}}=\overline{\beta_{1}}+\left(R^{\mathrm{ef}}\right)^{\mathrm{tr}} \overline{Q^{-1} P}-\overline{P^{\mathrm{tr}} Q^{-1} P} .
\end{gathered}
$$

Отметим, что наличие взаимодействия полей привело к появлению поправки к теплоемкости $\delta C$. 
Нахождение эффективных значений оставшихся коэффициентов никаких сложностей не вызывает, поэтому выпишем только эффективное значение еще одного упругого коэффициента

$$
c_{11}^{\mathrm{eff}}=\overline{c_{11}}+\left(R^{\mathrm{ef}}\right)^{\mathrm{tr}} \overline{Q^{-1} R}-\overline{R^{\mathrm{tr}} Q^{-1} R} .
$$

На основе полученных формул были построены графики зависимости пироэлектрического $p_{3}$ коэффициента и неаддитивной части эффективной теплоемкости $\delta C$ от толщины слоев (рис. 2,3). Первый слой - это титанат бария $\mathrm{BaTiO}_{3}$, второй $-\mathrm{CoFe}_{2} \mathrm{O}_{4}$, а третий - PZT-керамика. Данные для расчетов были взяты в $[10,17,18]$. Из графиков видно, что зависимость пирокоэффициента $p_{3}$ является монотонной, а добавка к теплоемкости для двуслойной системы имеет четко выраженный максимум при толщине слоя $\mathrm{BaTiO}_{3}$, в 11 раз превосходящую толщину $\mathrm{CoFe}_{2} \mathrm{O}_{4}$. Аналогичный максимум имеется и для трехслойной системы.

\section{Заключение}

Обсудим полученные результаты. Естественно, что при отсутствии взаимодействия полей выведенные выше формулы упрощаются и переходят в хорошо известные формулы для эффективных значений электрических [19], тепловых [20] и упругих [3] коэффициентов. Величины $\left\{\rho, \lambda_{1}, p_{1}, q_{1}, 2 c_{66}=c_{11}-c_{12}\right\}$ являются аддитивными. Для $\rho$ - это было ясно с самого начала, исходя из физического смысла рассматриваемой величины: масса системы слоев равна сумме масс каждого из слоев. Оставшиеся четыре аддитивных коэффициента характеризуют тепловые свойства в плоскости симметрии. Некоторые величины являются независимыми: их эффективные значения определяются только ими самими и не зависят от значений других величин в слоях. К таким относятся $\left\{\rho, \lambda_{1}, p_{1}, q_{1}, c_{44}, c_{66}\right\}$. Для других величин эффективные значения зависят от остальных коэффициентов. Например, теплоемкость $C$ и упругие константы $\left\{c_{11}, c_{12}\right\}$ зависят от значений еще 9 величин. Единственным упругим коэффициентом, который не зависит от термоэлектромагнитных свойств слоев, является $c_{44}$. Его эффективные значения одинаковы как в обычной теории упругости [3] при учете пьезоэлектрических свойств слоев [4], так и при учете тепловых явлений.

Основную роль при усреднении играет матрица $Q$. Ее элементы, характеризующие свойства слоя вдоль оси симметрии, взаимно влияют друг на друга. Величина этого влияния определяется значениями внедиагональных элементов $Q$. Тепловые характеристики слоев дают вклад только в значения упругих констант $c_{11}$ и $c_{12}$ и не оказывают влияния на электрические, магнитные и магнитоэлектрические коэффициенты. Наоборот, электромагнитоупругие коэффициенты принимают участие в усреднении пирокоэффициентов. В частности, даже при отсутствии пиросвойств у отдельных слоев они могут появиться в слоистой системе. Ситуация аналогична магнитоэлектрическому эффекту. В слоистых мультиферроиках в отдельно взятом слое его нет, тем не менее благодаря взаимодействию электрического и магнитного полей с упругим, вся структура обладает магнитоэлектрическим эффектом [5]. Данная аналогия дает возможность появления композитных материалов со значительными пирокоэффициентами.

К главным результатам работы следует отнести неаддитивность теплоемкости, описываемую двумя последними слагаемыми в (24). Эффективная теплоемкость для термоэлектромагнитных слоев не является взвешенным средним теплоемкостей отдельных слоев. Вызвано это тем обстоятельством, что тепло, переданное системе, преобразуется в упругую, электрическую и магнитную энергии. И это преобразование в разных слоях осуществляется по-разному. В результате возникает возможность управлять теплоемкостью, изменяя коэффициенты матрицы $Q$, например $\varepsilon_{33}$. Иначе говоря, теплоемкость системы получает дополнительную зависимость от электрического, магнитного или упругого поля. Для сегнетоэлектриков такая зависимость хорошо известна [21], но для слоистых структур из мультиферроиков выведена впервые. По аналогии с техникой СВЧ [22], можно вводить новую характеристику - управляемость теплоемкости. Отличие теплоемкости от взвешенной средней имеет место не только для слоев мультиферроиков, но и обычных диэлектриков и магнетиков. Правда, величина эффекта в этом случае ожидается весьма малой. Наиболее заметным эффект изменения теплоемкости будет именно для мультиферроиков, где взаимодействие полей различной природы играет важную роль.

Для того чтобы тепловые характеристики слоев повлияли, например, на электрические, необходимо рассмотреть более сложные уравнения, чем (10). В этих уравнениях должен присутствовать градиент температуры не только в уравнении теплопроводности, но и в других. Такая возможность реализуется при учете термополяризационного эффекта [23]. При этом эффекте в поляризации имеется слагаемое, пропорциональное градиенту температуры. В этом случае матрица $Q$ имеет размер $4 \times 4$, т. е. учитывает взаимодействие всех четырех полей.

Работа выполнена при государственной финансовой поддержке ведущих университетов Российской Федерации (субсидия 074-U01).

\section{Список литературы}

[1] Sanchez-Palencia E. Non homogeneous media and vibration theory. Lecture notes in physics. Berlin: Springer, 1980. 127 p.

[2] Bakhvalov N.S., Panasenko G.P. Homogenisation: averaging processes in periodic media: mathematical problems in the mechanics of composite materials. Dordrecht: Kluwer Academic Publishers, 2012. 366 p. 
[3] Молотков Л.А. Исследование распространения волн в пористых и трещиноватых средах на основе эффективных моделей Био и слоистых сред. СПб.: Наука, 2001. 348 с.

[4] Старков А.С., Старков И.А. // ЖЭТФ. 2014. Т. 146. Вып. 5(11). С. 980-989.

[5] Nan C.W., Bichurin M.I., Dong S., Viehland D., Srinivasan G. // J. Appl. Phys. 2008. Vol. 103(3). P. 031101-01031101-35.

[6] Ma J., Hu J., Li Z., Nan C.W. // Adv. Mater. 2011. Vol. 23. N 9. P. $1062-1087$.

[7] Пятаков А.П., Звездин А.К. // УФН. 2012. Т. 182. № 6. C. 593-620.

[8] Bichurin M.I., Viehland D. Magnetoelectricity in composites. Singapore: Pan Stanford Pub., 2011. 275 p.

[9] Starkov I.A., Starkov A.S. // Sol. Stat. Commun. 2016. Vol. 226. N 10. P. 5-7.

[10] Li J. // Mech. Mater. 2004. Vol. 36. N 10. P. 949-958.

[11] Вендик И.В., Вендик О.Г. // ЖТФ. 2013. Т. 83. Вып. 1. C. 3-28.

[12] Алешин В.И. //ЖТФ. 2007. Т. 77. Вып. 9. С. 54-60.

[13] Nazaikinskii A., Shatalov V.E., Sternin B.I. Methods of Noncommutative Analysis: Theory and Applications. Berlin: Walter de Gruyter, 1996. P. 74.

[14] Starkov I.A., Starkov A.S. // J. Nanophotonics. 2016. Vol . 10. N 3. P. 033503-033503.

[15] Lord H.W., Shulman Y. // J. Mech. Phys. Sol. 1967. Vol. 15. N 5. P. 299-309.

[16] Chandrasekharaiah D.S. // Acta Mech. 1988. Vol. 71. N 1-4. P. 39-49.

[17] Kondaiah P., Shankar K., Ganesan N. // Coupled Systems Mechanics. 2013. Vol. 2. N 1. P. 1-22.

[18] Zgonik M., Bernasconi P., Duelli M., Schlesser R., Günter P., Garrett M.H., Rytz D., Zhu Y., Wu X. // Phys. Rev. B. 1994. Vol. 50. N 9. P. 5941-5949.

[19] Рытов СМ. // ЖЭТФ. 1955. Т. 29. Вып. 5. С. 605-616.

[20] Дульнев Г.Н. Тепло- и массообмен в радиоэлектронной аппаратуре. М.: Высшая школа, 1984. 247 с.

[21] Старков А.С, Пахомов О.В., Старков И.А. // Письма в ЖЭТФ. 2010. Т. 91. Вып. 10. С. 556-560.

[22] Вендик О.Г. // ФТТ. 2009. Т. 51. Вып. 7. С. 1441-1446.

[23] Холкин А.Л., Трепаков В.А., Смоленский Г.А. // Письма ЖЭТФ. 1982. Т. 32. Вып. 3. С. 103-106. 